\title{
Transparent handpiece costs
}

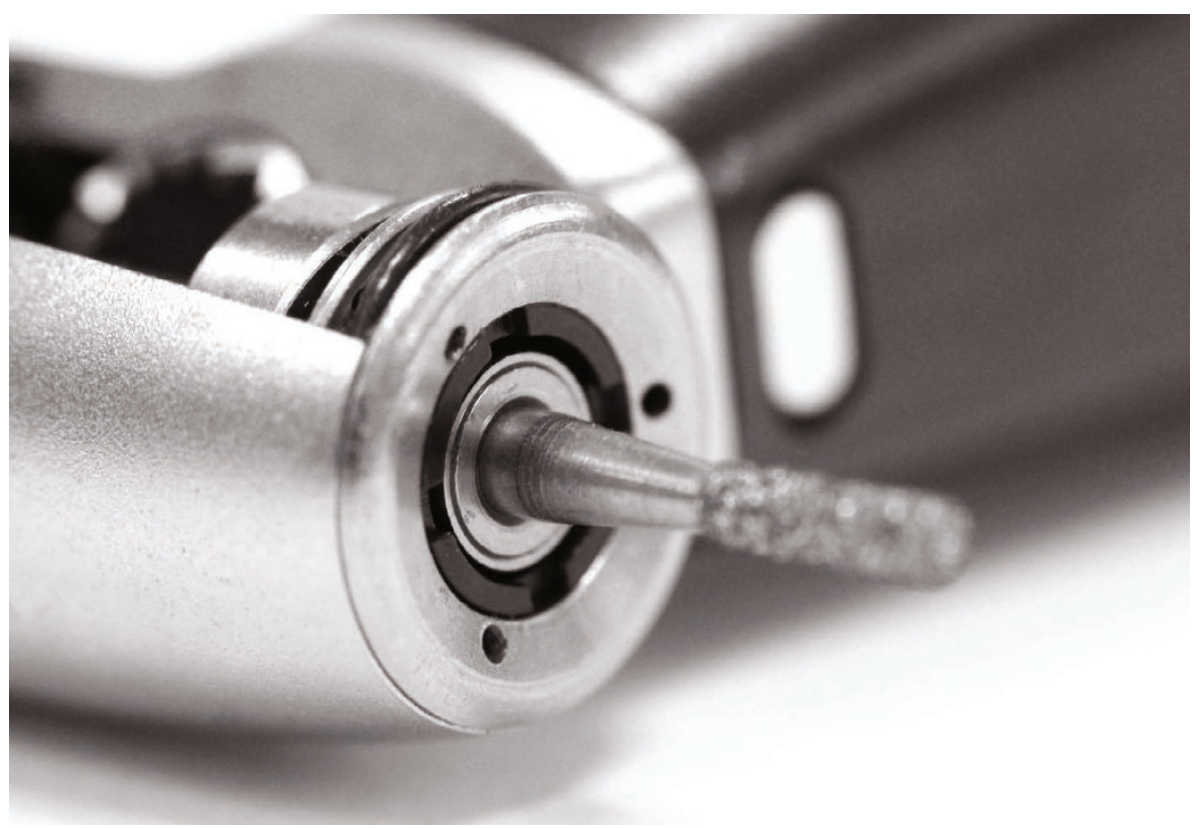

B.A. International, the UK's leading handpiece repair and supply house, offers its 36-month handpiece subscription solution to all practices wanting total handpiece cost transparency and an all-inclusive price with no hassle along the way.

Under this scheme, one simple fixed monthly payment of as little as $£ 51.10$ + VAT provides any practice with a minimum of three fibre optic turbines, LED coupling and ongoing maintenance oil, while also covering any potential handpiece repairs for a period of 36 months. Any number of additional turbines or contra-angles can be

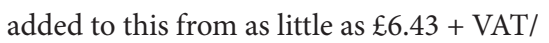
month. Even the BA DAC, the machine that automatically cleans, maintains and autoclaves handpieces can be subscribed to in this way - and that includes all consumables.

This subscription is also in most cases very tax efficient.

As B.A. International is certified to EN ISO 13485:2012 (the quality management system for medical devices), practitioners know that their handpieces are taken care of in a manner worthy of medical devices.

B.A. International thus offers practices a handpiece care solution that is guaranteed to be simple, efficient and completely hassle free.

Want to know more? Contact B.A.

International on 01604777700 and their friendly staff will be able to advise you.

\section{Armed ready for attack}

For superior protection from hacking, intrusion and cybercrime, CS Managed Service from Carestream Dental is a security-rich solution.

CS Managed Service offers top-notch firewalls and anti-virus protection, plus automatic updates and backups. The servers are housed in disaster-resistant facilities in multiple locations, with 24-7 monitoring of every account.

Cyber security should be on the agenda of every modern, efficient practice. If you do not protect your data adequately, you risk a fine from regulatory agencies and the threat of a potential civil law suit from patients who have had their personal information compromised.

While the problem is being fixed, your systems would need to be shut down, meaning you might be forced to cancel appointments and turn patients away.

CS Managed Service is just one solution from Carestream Dental, who specialise in helping practices run efficiently with its cutting-edge and creative practice management software.

Don't take any chances with sensitive data. Invest in the best. For more information contact Carestream Dental on 0800169 9692 or visit www.carestreamdental.co.uk.

\section{Fight the fear of change}

Are you happy with the products you use, or do you ever wonder if there are better out there?

Rolling orders, assumed patient preference, unfamiliarity with other brands - for the busy practitioner, there are many reasons to stick with the tried and tested.

But do not let barriers to change hold you back from sampling some of the amazing, high-quality tools and adjuncts now available on the market.

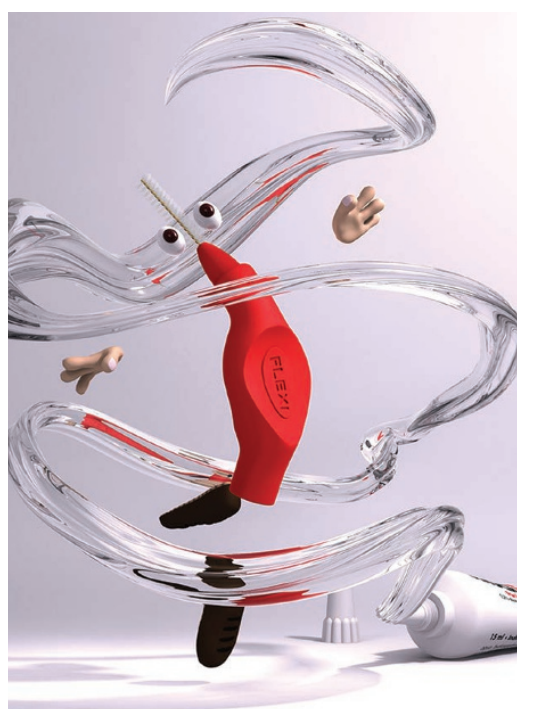

Tandex is a Danish company passionate about oral health and has developed a top-quality range for all your patients' needs. Its interdental brushes have evolved from collaboration with clinicians and leading technicians and offer accurate, gentle cleaning that is truly unparalleled.

The range, including the FLEXI brush, will complement regular brushing by easily removing debris from all the nooks and crannies. The unique features of the FLEXI include a longer wire for improved access, soft filaments and a wider head.

Don't let a fear of change hold you back! Click today, request samples and see what your patients think about Tandex.

The Tandex FLEXI is available from Survival 32 and other leading wholesalers.

For more information on Tandex's range of products, visit www.tandex.dk. 